\title{
Solving a stochastic heat equation driven by a bi-fractional noise
}

\author{
Xianye $Y u^{1}$, Xichao Sun ${ }^{2 *}$ and Litan Yan ${ }^{1,3}$
}

\section{"Correspondence:}

sunxichao626@126.com

${ }^{2}$ Department of Mathematics and

Physics, Bengbu University, 1866

Caoshan Rd., Bengbu, 233030,

P.R. China

Full list of author information is

available at the end of the article

\begin{abstract}
In this paper, we consider a stochastic heat equation with multiplicative bi-fractional Brownian sheet. Using the technique of Feynman-Kac formula and Malliavin calculus, we give an explicit formula of the weak solution and study the regularity.
\end{abstract}

MSC: $60 \mathrm{G} 15 ; 60 \mathrm{H} 05 ; 60 \mathrm{G} 17$

Keywords: Bi-fractional Brownian motion; heat equation; Malliavin calculus

\section{Introduction}

In recent years, there has been considerable interest in studying fractional Brownian motion $(\mathrm{fBm})$ due to its interesting properties and wide applications in various scientific areas such as turbulence, telecommunications, finance, and image processing. Some surveys and complete literatures for fBm can be found in Alós et al. [1], Biagini et al. [2], Decreusefond and Üstünel [3], Gradinaru et al. [4], Hu [5], Mishura [6], Nourdin [7], Nualart [8], Tudor [9], and the references therein. On the other hand, many authors have proposed to use more general self-similar Gaussian processes and random fields as stochastic models. Such applications have raised many interesting theoretical questions about selfsimilar Gaussian processes and fields in general. Therefore, some generalizations of the fBm have been introduced. However, in contrast to the extensive studies on $\mathrm{fBm}$, there has been little systematic investigation on other self-similar Gaussian processes. The main reason is the complexity of dependence structures for self-similar Gaussian processes that do not have stationary increments. Therefore, it seems interesting to study some extensions of $\mathrm{fBm}$.

The bi-fractional Brownian motion $B^{H, K}$ with indices $H \in(0,1)$ and $K \in(0,1]$ is an extension of $\mathrm{fBm}$ with Hurst index $H \in(0,1)$, which was first introduced by Houdré and Villa [10]. The bi-fBm $B^{H, K}$ with indices $H \in(0,1)$ and $K \in(0,1]$ is a zero-mean Gaussian process $B=\left\{B_{t}, t \in \mathbb{R}\right\}$ such that $B_{0}=0$ and

$$
E\left[B_{t}^{H, K} B_{s}^{H, K}\right]=R_{H, K}(t, s):=\frac{1}{2^{K}}\left[\left(|t|^{2 H}+|s|^{2 H}\right)^{K}-|t-s|^{2 H K}\right]
$$

Clearly, if $K=1$, then the process is an fBm with Hurst parameter $H$. The process $B$ is $H K$ selfsimilar, but it has no stationary increments. It has Hölder-continuous paths of order $\delta<H K$, and its paths are not differentiable.

(c) 2016 Yu et al. This article is distributed under the terms of the Creative Commons Attribution 4.0 International License (http://creativecommons.org/licenses/by/4.0/), which permits unrestricted use, distribution, and reproduction in any medium, provided you give appropriate credit to the original author(s) and the source, provide a link to the Creative Commons license, and indicate if changes were made. 
Definition 1.1 A bi-fractional noise with parameters $H, H^{\prime} \in(0,1), K, K^{\prime} \in(0,1]$ is a Gaussian random field $B=\left\{B_{t x}, t \geq 0, x \in \mathbb{R}\right\}$ with $B_{00}=0, E B_{t x}=0$, and

$$
E\left[B_{t x} B_{s y}\right]=R_{H, K}(t, s) R_{H^{\prime}, K^{\prime}}(x, y)
$$

for all $t, s \geq 0$. Moreover, the bi-fractional white noise with parameters $H$ and $K$ is a bifractional noise with parameters $H, K$ and $H^{\prime}=\frac{1}{2}, K^{\prime}=1$.

In order to expound our aim in this paper, we recall a classical result. Consider the stochastic heat equation

$$
\left\{\begin{array}{l}
\frac{\partial u}{\partial t}(t, x)=\Delta u(t, x)+\alpha(t, x) u(t, x), \\
u(0, x)=\varphi(x)
\end{array}\right.
$$

where $(t, x) \in[0, \infty) \times \mathbb{R}^{d}, \alpha(t, x)$ is a continuous function on $[0, \infty) \times \mathbb{R}^{d}$, and $\varphi$ is a bounded measurable function. Let $W_{t}^{x}=W_{t}+x$ be a $d$-dimensional Brownian motion starting from the point $x$. Then we can get the following Feynman-Kac formula (see Freidlin [11]) for the solution of stochastic heat equation (1.1):

$$
u(t, x)=E\left[\varphi\left(W_{t}^{x}\right) \exp \left\{\int_{0}^{t} \alpha\left(t-s, W_{s}^{x}\right) d s\right\}\right]
$$

In this paper, we extend the Feynman-Kac formula to the stochastic heat equation driven by a bi-fractional noise:

$$
\left\{\begin{array}{l}
\frac{\partial u}{\partial t}(t, x)=\Delta u(t, x)+\left(\frac{\partial^{2}}{\partial t \partial x} B(t, x)\right) u(t, x) \\
u(0, x)=\varphi(x)
\end{array}\right.
$$

where $B$ is a bi-fractional noise with parameters $H, K, H^{\prime}, K^{\prime}$ such that $H K>\frac{1}{2}, H^{\prime} K^{\prime}>\frac{1}{2}$, the stochastic integral is the Stratonovich integral, and $\varphi$ is a bounded measurable function. The difference between (1.1) and (1.3) is that $\frac{\partial^{2}}{\partial t \partial x} B(t, x)$ is a generalized random function, no longer a function of $x$ and $t$. Denoting by $E^{W}$ the expectation with respect to the Brownian motion $W_{t}^{x}$, we can formally rewrite the Feynman-Kac formula for the equation (1.3):

$$
u(t, x)=E^{W}\left[\varphi\left(W_{t}^{x}\right) \exp \left\{\int_{0}^{t} \int_{\mathbb{R}} \delta\left(W_{t-r}^{x}-y\right) B(d r, d y)\right\}\right],
$$

where $\delta$ denotes the Dirac delta function. The aim of this paper is to show that the process $u(t, x)$ given by $(1.4)$ is a weak solution of (1.3).

If $K=1$, then the process $B$ is a fractional Brownian sheet, and the questions stated were first studied by $\mathrm{Hu}$ et al. $[12,13]$. If $K \neq 1$, then this process is not a fractional Brownian sheet, and the questions stated were not studied and are not trivial. The main difficulty consists in the complexity of the dependence structure of a self-similar Gaussian process with nonstationary increments that does not have a representation based on the Wiener integral. This paper is organized as follows. In Section 2, we present some preliminaries for 
the bi-fractional noise. In Section 3, we show that the stochastic Feynman-Kac functional defined by

$$
V(t, x)=\int_{0}^{t} \int_{\mathbb{R}} \delta\left(W_{t-r}^{x}-y\right) B(d r, d y)
$$

is well defined and exponentially integrable by using a suitable approximation of the Dirac delta function under some suitable conditions. In Section 4, we show that the process (1.4) is a weak solution to equation (1.3). In Section 5, we study the regularity of the weak solution. We show that the solution is Hölder continuous and the probability law of the solution admits a smooth density with respect to the Lebesgue measure.

\section{Preliminaries}

In this section, we briefly recall the definition and properties of the stochastic integral with respect to a bi-fractional noise. As for a Gaussian process, we can construct a stochastic calculus of variations with respect to $B$. We refer to Alós et al. [1] and Nualart [8] for a complete description of stochastic calculus with respect to Gaussian processes. Here we only recall the basic elements of this theory (see Es-sebaiy and Tudor [14]). More works on bi-fBm can be found in Jiang and Wang [15], Kruk et al. [16], Lei and Nualart [17], Russo and Tudor [18], Tudor and Xiao [19], Shen and Yan [20], Yan et al. [21, 22], and the references therein.

As we pointed out before, a bi-fractional noise $B=\left\{B_{t x}, 0 \leq t \leq T, x \in \mathbb{R}\right\}$ on a probability space $(\Omega, \mathscr{F}, P)$ with indices $H, H^{\prime} \in(0,1)$ and $K, K^{\prime} \in(0,1]$ is a rather special class of selfsimilar Gaussian random fields such that $B_{00}=0$ and

$$
E\left[B_{t x} B_{s y}\right]=R_{H, K}(t, s) R_{H^{\prime}, K^{\prime}}(x, y)
$$

where

$$
R_{H, K}(t, s)=\frac{1}{2^{K}}\left[\left(|t|^{2 H}+|s|^{2 H}\right)^{K}-|t-s|^{2 H K}\right] .
$$

In other words, $B$ is a bi-fractional Brownian sheet with Hurst parameters $H$ and $K$ in the time variable and $H^{\prime}$ and $K^{\prime}$ in the space variable. Throughout this paper, we assume that $2 H K, 2 H^{\prime} K^{\prime} \geq 1$.

Let $\mathcal{H}$ be the completion of the linear space $\mathcal{E}$ generated by the indicator functions $1_{[0, t]}$, $t \in[0, T]$, with respect to the inner product

$$
\left\langle 1_{[0, s] \times[0, x]}, 1_{[0, t] \times[0, y]}\right\rangle_{\mathcal{H}}=R_{H, K}(t, s) R_{H^{\prime}, K^{\prime}}(x, y),
$$

where we assume that $1_{[0, x]}=-1_{[x, 0]}$ if $x<0$. The mapping $\psi \in \mathcal{E} \rightarrow B(\psi)$ is an isometry from $\mathcal{E}$ to the Gaussian space generated by $B$, and it can be extended to $\mathcal{H}$. We will denote this isometry by

$$
B(\psi)=\int_{0}^{\infty} \int_{\mathbb{R}} \psi(t, x) B(d t, d x)
$$

for $\psi \in \mathcal{H}$. For $\varphi, \psi \in \mathcal{E}$, we have

$$
E[B(\varphi) B(\psi)]=\langle\varphi, \psi\rangle_{\mathcal{H}}=\kappa \int_{\mathbb{R}_{+}^{2} \times \mathbb{R}^{2}} \varphi(s, x) \psi(t, y) \zeta_{H, K}(s, t) \zeta_{H^{\prime}, K^{\prime}}(x, y) d s d t d x d y
$$


with a constant $\kappa>0$ depending only on $H, K, H^{\prime}, K^{\prime}$, where

$$
\zeta_{\alpha, \beta}(s, t)=|t-s|^{2 \alpha \beta-2}
$$

with $\alpha \beta>\frac{1}{2}$, and

$$
\zeta_{\alpha, \beta}(s, t)=\left(|s|^{2 \alpha}+|t|^{2 \alpha}\right)^{\beta-2}|s t|^{2 \alpha-1}
$$

with $\alpha \beta=\frac{1}{2}$. Moreover, $\mathcal{H}$ denotes the class of measurable functions $\psi$ on $\mathbb{R}_{+} \times \mathbb{R}$ satisfying

$$
\int_{\mathbb{R}_{+}^{2} \times \mathbb{R}^{2}} \varphi(s, x) \psi(t, y) \zeta_{H, K}(s, t) \zeta_{H^{\prime}, K^{\prime}}(x, y) d s d t d x d y<\infty
$$

Let us denote by $\mathcal{S}$ the set of smooth functionals of the form

$$
F=f\left(B\left(\psi_{1}\right), B\left(\psi_{2}\right), \ldots, B\left(\psi_{n}\right)\right)
$$

where $f \in C_{b}^{\infty}\left(\mathbb{R}^{n}\right)$ and $\psi_{i} \in \mathcal{H}$. The Malliavin derivative $D^{B}$ of a functional $F$ as before is given by

$$
D^{B} F=\sum_{j=1}^{n} \frac{\partial f}{\partial x_{j}}\left(B\left(\psi_{1}\right), B\left(\psi_{2}\right), \ldots, B\left(\psi_{n}\right)\right) \psi_{j} .
$$

The derivative operator $D^{B}$ is then a closable operator from $L^{2}(\Omega)$ into $L^{2}(\Omega ; \mathcal{H})$. We denote by $\mathbb{D}^{1,2}$ the closure of $\mathcal{S}$ with respect to the norm

$$
\|F\|_{1,2}:=\sqrt{E|F|^{2}+E\left\|D^{B} F\right\|_{\mathcal{H}}^{2}} .
$$

The divergence integral $\delta^{B}$ is the adjoint of the derivative operator $D^{B}$ given by the duality relationship

$$
E\left[F \delta^{B}(u)\right]=E\left\langle D^{B} F, u\right\rangle_{\mathcal{H}}
$$

for any element $F \in \mathbb{D}^{1,2}$ and any $u \in L^{2}(\Omega ; \mathcal{H})$ in $\delta^{B}$. A random variable $u \in L^{2}(\Omega ; \mathcal{H})$ belongs to the domain of the divergence operator $\delta^{B}$, denoted by $\operatorname{Dom}\left(\delta^{B}\right)$, if

$$
E\left|\left\langle D^{B} F, u\right\rangle_{\mathcal{H}}\right| \leq c\|F\|_{L^{2}(\Omega)}
$$

for every $F \in \mathbb{D}^{1,2}$, where $c$ is a constant depending only on $u$. We have also the following formula:

$$
F B(\psi)=\delta^{B}(F \psi)+\left\langle D^{B} F, \psi\right\rangle_{\mathcal{H}}
$$

for any $\psi \in \mathcal{H}$ and any random variable $F \in \mathbb{D}^{1,2}$. The operator $\delta^{B}$ is also called the Skorokhod integral. The readers can refer to Nualart [8] for a detailed account of the Malliavin calculus with respect to a Gaussian process. If $u$ and $D^{B} F$ are almost surely measurable functions on $\mathbb{R}_{+} \times \mathbb{R}$ satisfying condition (2.2), then the duality formula (2.3) can be writ- 
ten using the expression of the inner product in $\mathcal{H}$ :

$$
E\left[\delta^{B}(u) F\right]=\kappa \int_{\mathbb{R}_{+}^{2} \times \mathbb{R}^{2}}\left(D_{s, x}^{B} F\right) u(t, y) \zeta_{H, K}(s, t) \zeta_{H^{\prime}, K^{\prime}}(x, y) d s d t d x d y .
$$

\section{The stochastic Feynman-Kac functional}

Let $W=\left\{W_{t}, t \geq 0\right\}$ be a standard Brownian motion independent of $B$, and $W^{x}=W+x$. In this section, we study the stochastic Feynman-Kac functional

$$
V(t, x):=\int_{0}^{t} \int_{\mathbb{R}} \delta\left(W_{t-r}^{x}-y\right) B(d r, d y)
$$

where $\delta$ denotes the Dirac delta function. We denote by $E^{W}(\Psi(B, W))$ (resp., $E^{B}(\Psi(B, W))$ ) the expectation of a functional $\Psi(B, W)$ with respect to $W$ (resp., with respect to $B$ ). We use $E$ to denote the composition $E^{B} E^{W}$, which is a random variable depending only on $B$ or $W$.

For any $\varepsilon>0$ and $\tau>0$, we define the functions $p_{\varepsilon}(x)$ and $\phi_{\tau}(t)$ by

$$
p_{\varepsilon}(x)=\frac{1}{\sqrt{2 \pi \varepsilon}} e^{-\frac{x^{2}}{2 \varepsilon}} \equiv \frac{1}{2 \pi} \int_{\mathbb{R}} e^{i x \xi} e^{-\varepsilon \frac{\xi^{2}}{2}} d \xi, \quad x \in \mathbb{R},
$$

and

$$
\phi_{\tau}(t)=\frac{1}{\tau} 1_{[0, \tau]}(t), \quad t \geq 0 .
$$

Then $\phi_{\tau}(t) p_{\varepsilon}(x)$ is an approximation of the Dirac delta function $\delta(t, x)$ as $\varepsilon$ and $\tau$ tend to zero.

Lemma 3.1 Let $\zeta_{H, K}$ be defined in Section 2 , and let $W=\left\{W_{t}, t \geq 0\right\}$ be a standard Brownian motion starting at zero. Then we have

$$
E\left[\zeta_{H, K}\left(W_{t}, W_{s}\right)\right] \leq \frac{C s^{2 H-1}}{(t-s)^{1-H}}
$$

for all $t>s>0$.

Proof Recall that if $\left(G_{1}, G_{2}\right)$ is a Gaussian couple, then we can write

$$
G_{2}=\frac{\operatorname{Cov}\left(G_{1}, G_{2}\right)}{\operatorname{Var}\left(G_{1}\right)} G_{1}+\sqrt{\operatorname{Var}\left(G_{2}\right)-\frac{\operatorname{Cov}^{2}\left(G_{1}, G_{2}\right)}{\operatorname{Var}\left(G_{1}\right)} \eta},
$$

where $\eta$ is a standard normal random variable independent of $G_{1}$, and $\operatorname{Var}(\cdot)$ denotes the variance. We then can write

$$
W_{t}=\sqrt{s} \xi+\sqrt{t-s} \eta
$$

in distribution, where $\xi$ and $\eta$ are two independent standard normal random variables, which implies that

$$
\zeta_{H, K}\left(W_{t}, W_{s}\right)=\frac{|(\sqrt{s} \xi+\sqrt{t-s} \eta) \sqrt{s} \xi|^{2 H-1}}{\left(|\sqrt{s} \xi+\sqrt{t-s} \eta|^{2 H}+|\sqrt{s} \xi|^{2 H}\right)^{2-K}}
$$


in distribution. Thus, an elementary calculation shows that

$$
E\left(\frac{|(\sqrt{s} \xi+\sqrt{t-s} \eta) \sqrt{s} \xi|^{2 H-1}}{\left(|\sqrt{s} \xi+\sqrt{t-s} \eta|^{2 H}+|\sqrt{s} \xi|^{2 H}\right)^{2-K}}\right) \leq \frac{C s^{2 H-1}}{(t-s)^{1-H}}
$$

for all $0<s<t$.

Lemma 3.2 Let $\zeta_{H, K}$ be defined in Section 2. For all $H, K \in(0,1)$ and $2 H K \geq 1$, we have

$$
\begin{aligned}
& \int_{\mathbb{R}^{2}} p_{\varepsilon}(x+u) p_{\varepsilon^{\prime}}(y+v) \zeta_{H, K}(u, v) d u d v \leq C \zeta_{H, K}(x, y) \text { and } \\
& \int_{[0, t]^{2}} \phi_{\varepsilon}(t-s-u) \phi_{\varepsilon^{\prime}}(t-r-v) \zeta_{H, K}(u, v) d u d v \leq C \zeta_{H, K}(t-s, t-r)
\end{aligned}
$$

for all $\varepsilon, \varepsilon^{\prime}>0, s, r \in[0, t]$, and $x, y \in \mathbb{R}$.

Proof Let $H K>\frac{1}{2}$, and let $\xi$ be a standard normal random variable. We then have (see $\mathrm{Hu}$ et al. [12])

$$
E|x+\varepsilon \xi|^{-\alpha} \leq C \min \left\{\varepsilon^{-\alpha}, x^{-\alpha}\right\}
$$

for $0<\alpha<1, \varepsilon, x>0$. As a corollary, we have

$$
\begin{aligned}
& \int_{\mathbb{R}^{2}} p_{\varepsilon}(x+u) p_{\varepsilon^{\prime}}(y+v) \zeta_{H, K}(u, v) d u d v \\
& \quad=E\left|\varepsilon \xi-x-\varepsilon^{\prime} \eta+y\right|^{2 H K-2} \leq C|x-y|^{2 H^{\prime} K^{\prime}-2} .
\end{aligned}
$$

Similarly, we also get (3.4).

Let now $H K=\frac{1}{2}$. Then we have

$$
\begin{aligned}
& \int_{\mathbb{R}^{2}} p_{\varepsilon}(x+u) p_{\varepsilon^{\prime}}(y+v) \zeta_{H, K}(u, v) d u d v \\
& \quad=\int_{\mathbb{R}^{2}} p_{\varepsilon}(x+u) p_{\varepsilon^{\prime}}(y+v) \frac{|u v|^{2 H-1} d u d v}{\left(|u|^{2 H}+|v|^{2 H}\right)^{2-K}} \\
& \quad=E\left[\frac{\left|(\varepsilon \xi-x)\left(\varepsilon^{\prime} \eta-y\right)\right|^{2 H-1}}{\left(|\varepsilon \xi-x|^{2 H}+\left|\varepsilon^{\prime} \eta-y\right|^{2 H}\right)^{2-K}}\right] \leq C \zeta(x, y) .
\end{aligned}
$$

On the other hand, for $H K=\frac{1}{2}$, we have

$$
p_{\varepsilon}(x) \geq p_{\varepsilon}(x) 1_{[0, \sqrt{\varepsilon}]}(x)=\frac{1}{\sqrt{2 \pi \varepsilon}} e^{-\frac{x^{2}}{2 \varepsilon}} 1_{[0, \sqrt{\varepsilon}]}(x) \geq \frac{1}{\sqrt{2 \pi e}} \phi_{\sqrt{\varepsilon}}(x)
$$

for all $\varepsilon>0$, which gives

$$
\begin{aligned}
& \int_{[0, t]^{2}} \phi_{\varepsilon}(t-s-u) \phi_{\varepsilon^{\prime}}(t-r-v) \zeta_{H, K}(u, v) d u d v \\
& \quad \leq C \int_{\mathbb{R}^{2}} p_{\varepsilon}(t-s-u) p_{\varepsilon^{\prime}}(t-r-v) \zeta_{H, K}(u, v) d u d v \leq C \zeta_{H, K}(t-s, t-r)
\end{aligned}
$$

for all $\varepsilon, \varepsilon^{\prime}>0$ and $s, r>0$. 
We obtain some approximations as follows:

$$
\begin{aligned}
& B^{\varepsilon, \tau}(t, x)=\int_{0}^{t} \int_{\mathbb{R}} \phi_{\tau}(t-s) p_{\varepsilon}(x-y) B(s, y) d s d y, \\
& A_{t, x}^{\varepsilon, \tau}(r, y)=\int_{0}^{t} \phi_{\tau}(t-s-r) p_{\varepsilon}\left(W_{s}^{x}-y\right) d s
\end{aligned}
$$

and

$$
V^{\varepsilon, \tau}(t, x)=\int_{0}^{t} \int_{\mathbb{R}} A_{t, x}^{\varepsilon, \tau}(r, y) B(d r, d y)
$$

for all $t, s, r \geq 0$ and $x, y \in \mathbb{R}$. Then

- $B^{\varepsilon, \tau}(t, x)$ is an approximation of the bi-fractional noise $B(t, x)$;

- $A_{t, x}^{\varepsilon, \tau}(r, y)$ is an approximation of the Dirac delta function $\delta\left(W_{t-r}^{x}-y\right)$.

Theorem 3.1 Suppose that $2 H K \geq 1,2 H^{\prime} K^{\prime} \geq 1$, and $2 H K+H^{\prime} K^{\prime}>2$. Then for any $\tau>0$ and $\varepsilon>0$, we have that $A_{t, x}^{\varepsilon, \tau}$ belongs to $\mathcal{H}$ and the family of random variables $V^{\varepsilon, \tau}(t, x)$ converges in $L^{2}$ to a limit denoted by

$$
V(t, x):=\int_{0}^{t} \int_{\mathbb{R}} \delta\left(W_{t-r}^{x}-y\right) B(d r, d y)
$$

which is called the stochastic Feynman-Kac functional. Conditional on $W, V(t, x)$ is a Gaussian random variable with mean 0 and variance

$$
\sigma^{2}(W)=\kappa \int_{0}^{t} \int_{0}^{t}|r-s|^{2 H K-2} \zeta_{H^{\prime}, K^{\prime}}\left(W_{r}, W_{s}\right) d r d s
$$

Proof Let $\varepsilon, \varepsilon^{\prime}, \tau, \tau^{\prime}>0$. Clearly, the condition $2 H K+H^{\prime} K^{\prime}>2$ implies that $2 H K>1$. To show that $A_{t, x}^{\varepsilon, \tau}$ belongs to the space $\mathcal{H}$ almost surely, we compute the inner product

$$
\begin{aligned}
0 \leq & \left\langle A_{t, x}^{\varepsilon, \tau},\left.A_{t, x}^{\varepsilon^{\prime}, \tau^{\prime}}\right|_{\mathcal{H}}=\kappa \int_{[0, t]^{4}} \int_{\mathbb{R}^{2}} p_{\varepsilon}\left(W_{s}^{x}-y\right) p_{\varepsilon^{\prime}}\left(W_{r}^{x}-z\right) \phi_{\tau}(t-s-u)\right. \\
& \cdot \phi_{\tau^{\prime}}(t-r-v)|u-v|^{2 H K-2} \zeta_{H^{\prime}, K^{\prime}}(y, z) d y d z d u d v d s d r \\
\leq & C \int_{[0, t]^{2}}|s-r|^{2 H K-2} \zeta_{H^{\prime}, K^{\prime}}\left(W_{s}, W_{r}\right) d s d r
\end{aligned}
$$

by Lemma 3.2.

For all $2 H^{\prime} K^{\prime}>1$ and $t \geq 0$, we have

$$
\begin{aligned}
E^{W}\left(\left\langle A_{t, x}^{\varepsilon, \tau}, A_{t, x}^{\varepsilon^{\prime}, \tau^{\prime}}\right\rangle_{\mathcal{H}}\right) & \leq C \int_{[0, t]^{2}}|s-r|^{2 H K-2} E \zeta_{H^{\prime}, K^{\prime}}\left(W_{s}, W_{r}\right) d s d r \\
& =C \int_{[0, t]^{2}}|s-r|^{2 H K-2} E\left|W_{s}-W_{r}\right|^{2 H^{\prime} K^{\prime}-2} d s d r \\
& =C t^{2 H K+H^{\prime} K^{\prime}-1} E\left(|\xi|^{2 H^{\prime} K^{\prime}-2}\right)<\infty
\end{aligned}
$$


where $\xi$ is a standard normal random variable. Similarly, for all $2 H^{\prime} K^{\prime}=1$ and $t \geq 0$, we also have

$$
E^{W}\left(\left\langle A_{t, x}^{\varepsilon, \tau}, A_{t, x}^{\varepsilon^{\prime}, \tau^{\prime}}\right\rangle_{\mathcal{H}}\right) \leq C \int_{0}^{t} \int_{0}^{t}|s-r|^{2 H K+H^{\prime}-3} d s d r<\infty
$$

by Lemma 3.1 .

Therefore, $A_{t, x}^{\varepsilon, \tau}$ belongs to the space $\mathcal{H}$ almost surely for all $\varepsilon>0$ and $\tau>0$, which implies that the random variables $V^{\varepsilon, \tau}(t, x)$ are well defined, and we get

$$
E^{W} E^{B}\left[V^{\varepsilon, \tau}(t, x) V^{\varepsilon^{\prime}, \tau^{\prime}}(t, x)\right]=E^{W}\left[\left\langle A_{t, x}^{\varepsilon, \tau}, A_{t, x}^{\varepsilon^{\prime}, \tau^{\prime}}\right\rangle_{\mathcal{H}}\right]
$$

It follows from the dominated convergence theorem that there exists a constant $C$ depending only on $t, H, K, H^{\prime} K^{\prime}$ such that

$$
E^{W} E^{B}\left[V^{\varepsilon, \tau}(t, x) V^{\varepsilon^{\prime}, \tau^{\prime}}(t, x)\right] \rightarrow C
$$

as $\varepsilon, \varepsilon^{\prime}, \tau, \tau^{\prime}$ tend to zero. This shows that

$$
E\left[\left|V^{\varepsilon, \tau}(t, x)-V^{\varepsilon^{\prime}, \tau^{\prime}}(t, x)\right|^{2}\right] \longrightarrow 0
$$

as $\varepsilon, \varepsilon^{\prime}, \tau, \tau^{\prime}$ tend to zero. As a consequence, $V^{\varepsilon, \tau}(t, x)$ converges in $L^{2}$ to a limit denoted by $V(t, x)$.

Finally, by a similar argument we obtain (3.9), and the proof is completed.

Now, we show the exponential integrability of the random variable $V(t, x)$ defined in Theorem 3.1.

Theorem 3.2 Let the random variable $V(t, x)$ be defined in Theorem 3.1. If $2 H K, 2 H^{\prime} K^{\prime} \geq 1$ and $2 H K+H^{\prime} K^{\prime}>2$, then we have

$$
E\left[e^{\lambda V(t, x)}\right]<\infty
$$

for any $\lambda \in \mathbb{R}$.

Proof Let $2 H K, 2 H^{\prime} K^{\prime} \geq 1$ and $2 H K+H^{\prime} K^{\prime}>2$. Then $2 H K>1$ and $2 H^{\prime} K^{\prime} \geq 1$. Denote

$$
\Lambda_{t}:=\int_{0}^{t} \int_{0}^{t}|r-s|^{2 H K-2} \zeta_{H^{\prime}, K^{\prime}}\left(W_{r}, W_{s}\right) d r d s
$$

and $\Theta_{t}=\sqrt{\Lambda_{t}}$ for all $t \geq 0$. Then $\Lambda_{t} \geq 0$ is nondecreasing and pathwise continuous. It follows from (3.9) and the scaling property of Brownian motion that

$$
\begin{aligned}
E\left[e^{\lambda V(t, x)}\right] & =E^{W} E\left[e^{\lambda V(t, x)} \mid W\right]=E^{W}\left[e^{\operatorname{Var}(\lambda V(t, x) \mid W)}\right] \\
& =E\left[\exp \left\{\lambda \int_{0}^{t} \int_{\mathbb{R}} \delta\left(W_{t-r}^{x}-y\right) B(d r, d y)\right\}\right] \\
& =E\left[\exp \left\{\frac{1}{2} \lambda^{2} \kappa \int_{0}^{t} \int_{0}^{t}|r-s|^{2 H K-2} \zeta_{H^{\prime}, K^{\prime}}\left(W_{r}, W_{s}\right) d r d s\right\}\right]
\end{aligned}
$$

for all $t \geq 0$. 
Case I: $2 H K, 2 H^{\prime} K^{\prime}>1$ and $2 H K+H^{\prime} K^{\prime}>2$. We have

$$
\begin{aligned}
E\left[e^{\lambda V(t, x)}\right] & =E\left[\exp \left\{\frac{1}{2} \lambda^{2} \kappa \int_{0}^{t} \int_{0}^{t}|r-s|^{2 H K-2}\left|W_{r}-W_{s}\right|^{2 H^{\prime} K^{\prime}-2} d r d s\right\}\right] \\
& =E\left[\exp \left\{\frac{1}{2} \lambda^{2} \kappa t^{2 H K+H^{\prime} K^{\prime}-1} \Lambda_{1}\right\}\right]
\end{aligned}
$$

for all $t \geq 0$. Then it suffices to show that the random variable $\Lambda_{1}$ has exponential moments of all orders. This will be done in two steps.

Step 1 . By the identity

$$
\left|B_{s}-B_{r}\right|^{\beta-2}=C \int_{\mathbb{R}}\left|B_{s}-x\right|^{\frac{\beta-3}{2}}\left|B_{r}-x\right|^{\frac{\beta-3}{2}} d x
$$

for all $1<\beta<2$ we have

$$
\Lambda_{t}=\int_{\mathbb{R}^{2}} \eta_{t}^{2}(u, x) d u d x
$$

where

$$
\eta_{t}=C \int_{0}^{t}|s-u|^{\frac{2 H K-3}{2}}\left|B_{s}-x\right|^{\frac{2 H^{\prime} K^{\prime}-3}{2}} d s
$$

Denote $\tilde{B}_{s}=B_{t+s}-B_{t}$ for all $t, s \geq 0$. Then we have

$$
\begin{aligned}
\tilde{\eta}_{t_{2}}(u, x) & :=\eta_{t_{1}+t_{2}}(u, x)-\eta_{t_{1}}(u, x)=C \int_{t_{1}}^{t_{1}+t_{2}}|s-u|^{\frac{2 H K-3}{2}}\left|B_{s}-x\right|^{\frac{2 H^{\prime} K^{\prime}-3}{2}} d s \\
& =C \int_{0}^{t_{2}}\left|s+t_{1}-u\right|^{\frac{2 H K-3}{2}}\left|\tilde{B}_{s}+B_{t_{1}}-x\right|^{\frac{2 H^{\prime} K^{\prime}-3}{2}} d s
\end{aligned}
$$

for $t_{1}, t_{2}>0$. It follows from triangular inequality and translation invariance that

$$
\begin{aligned}
\Theta_{t_{1}+t_{2}} & \leq \Theta_{t_{1}}+\left(\int_{\mathbb{R}^{2}}\left[\eta_{t_{1}+t_{2}}(u, x)-\eta_{t_{1}}(u, x)\right]^{2} d u d x\right)^{1 / 2} \\
& =\Theta_{t_{1}}+\tilde{\Theta}_{t_{2}}
\end{aligned}
$$

for $t_{1}, t_{2}>0$, where $\tilde{\Theta}_{t_{2}}$ is independent of $\left\{\Theta_{s}, 0 \leq s \leq t_{1}\right\}$ and has the same distribution as $\Theta_{t_{1}}$. Therefore, the process $\Theta_{t}$ is subadditive.

Step 2. By Theorem 1.3.5 in [23] we have

$$
E\left[e^{\theta \Theta t}\right]<\infty
$$

and

$$
\lim _{t \rightarrow \infty} \frac{1}{t} \log E\left[e^{\theta \Theta t}\right]=\Phi(\theta)=\Phi(1) \theta^{\frac{2}{2 H K+H^{\prime} K^{\prime}-1}}
$$


for any $\theta, t>0$, by the scaling property, where $0 \leq \Phi(\theta)<\infty$. It follows from the Chebyshev inequality that

$$
\limsup _{t \rightarrow \infty} \frac{1}{t} \log P\left(\Theta_{t} \geq t\right) \leq \Phi(\theta)-\theta=\Phi(1) \theta \frac{2}{\frac{2}{2 H K+H^{\prime} K^{\prime}-1}}-\theta
$$

for all $\theta>0$, which gives

$$
\limsup _{t \rightarrow \infty} \frac{1}{t} \log P\left(\Theta_{t} \geq t\right) \leq \min _{\theta>0}\left(\Phi(1) \theta \frac{2}{2 H K+H^{\prime} K^{\prime}-1}-\theta\right) .
$$

Hence, there exists $a>0$ such that

$$
\limsup _{t \rightarrow \infty} \frac{1}{t} \log P\left(\Theta_{t} \geq t\right) \leq-a
$$

and

$$
P\left(\Theta_{1} \geq t^{\frac{3-2 H K-H^{\prime} K^{\prime}}{2}}\right) \leq e^{-\frac{1}{2} a t}
$$

when $t>N$ for some $N>0$. Combining this with the fact that

$$
E \Psi(X)=E \int_{0}^{X} \phi(x) d x+\Phi(0)=\int_{0}^{\infty} \phi(x) P(X \geq x) d x+\phi(0)
$$

for $\Phi(y)=\int_{0}^{y} \phi(x) d x+\Phi(0)$ and all random variables $X \geq 0$, we get

$$
\begin{aligned}
E\left[\exp \left(\theta \Theta_{1}^{\frac{2}{2 H K+H^{\prime} K^{\prime}-1}}\right)\right] & =\int_{0}^{\infty} P\left(\theta \Theta_{1}^{\frac{2}{2 H K+H^{\prime} K^{\prime}-1}} \geq x\right) e^{x} d x+1 \\
& \leq \int_{0}^{N} e^{x} d x+\int_{N}^{\infty} P\left(\theta \Theta_{1}^{\frac{2}{2 H K+H^{\prime} K^{\prime}-1}} \geq x\right) e^{x} d x+1 \\
& \leq \sum_{k=N}^{\infty} P\left(\theta \Theta_{1}^{\frac{2}{2 H K+H^{\prime} K^{\prime}-1}} \geq k\right) e^{k+1}+e^{N} \leq \sum_{k=N}^{\infty} e^{-\frac{a k}{2 \theta}+k+1}+e^{N}
\end{aligned}
$$

for all $\theta<\frac{a}{4}$. This gives the critical integrability

$$
E\left[\exp \left(\theta \Theta_{1}^{\frac{2}{2 H K+H^{\prime} K^{\prime}-1}}\right)\right]<\infty
$$

which implies that $E\left[\exp \left(\lambda \Theta_{1}^{2}\right)\right]<\infty$ for all $\lambda>0$.

Thus, we have proved the theorem for $2 H K, 2 H^{\prime} K^{\prime}>1$ and $2 H K+H^{\prime} K^{\prime}>2$.

Case II: $2 H K>1,2 H^{\prime} K^{\prime}=1$ and $2 H K+H^{\prime} K^{\prime}>2$. We have

$$
E\left[e^{\lambda V(t, x)}\right]=E\left[\exp \left\{\frac{1}{2} \lambda^{2} \kappa \int_{0}^{t} \int_{0}^{t}|r-s|^{2 H K-2} \zeta_{H^{\prime}, K^{\prime}}\left(W_{r}, W_{s}\right) d r d s\right\}\right]
$$

for all $t \geq 0$. Now the proof follows similarly to Case I. 


\section{The Feynman-Kac formula}

In this section, we give the Feynman-Kac formula of equation (1.3). Let us first recall the definitions of the Stratonovich integral and weak solution to (1.3). For any $\varepsilon, \tau>0$, we define

$$
\dot{B}^{\varepsilon, \tau}(t, x)=\int_{0}^{t} \int_{\mathbb{R}} \phi_{\tau}(t-s) p_{\varepsilon}(x-y) B(d s, d y)
$$

To provide a notion of solution for the stochastic heat equation driven by bi-fractional sheet (1.3), we need the following definition of the Stratonovich integral, which is introduced by Russo and Vallois [24] and Hu et al. [12].

Definition 4.1 Let a random field $v=\{v(t, x), t \geq 0, x \in \mathbb{R}\}$ satisfy

$$
\int_{0}^{T} \int_{\mathbb{R}}|v(t, x)| d x d t<\infty
$$

almost surely for all $T>0$. We define the Stratonovich integral as

$$
\int_{0}^{T} \int_{\mathbb{R}} v(t, x) B(d t, d x):=\lim _{\varepsilon, \tau \downarrow 0} \int_{0}^{T} \int_{\mathbb{R}} v(t, x) \dot{B}^{\varepsilon, \tau}(t, x) d x d t
$$

if the limit exists in probability.

Definition 4.2 We say that a random field $u=\{u(t, x), t \geq 0, x \in \mathbb{R}\}$ is a weak solution of (1.3) if, for any $C^{\infty}$-function $f$ with compact support on $\mathbb{R}$, we have

$$
\begin{aligned}
\int_{\mathbb{R}} u(t, x) f(x) d x= & \int_{\mathbb{R}} f(x) \varphi(x) d x \\
& +\frac{1}{2} \int_{0}^{t} \int_{\mathbb{R}} u(s, x) \Delta f(x) d x d s+\int_{0}^{t} \int_{\mathbb{R}} u(s, x) f(x) B(d s, d x)
\end{aligned}
$$

almost surely for all $t \geq 0$.

Theorem 4.1 Let $2 H K, 2 H^{\prime} K^{\prime}>1,2 H K+H^{\prime} K^{\prime}>2$, and let $\varphi$ be a bounded measurable function. Then the process

$$
u(t, x)=E^{W}\left[\varphi\left(W_{t}^{x}\right) \exp \left\{\int_{0}^{t} \int_{\mathbb{R}} \delta\left(W_{t-r}^{x}-y\right) B(d r, d y)\right\}\right]
$$

is a weak solution to (1.3), where $E^{W}$ denotes the expectation with respect to the Brownian motion $W_{t}^{x}$, and $\delta$ denotes the Dirac delta function.

In order to prove the theorem, we need some preliminaries. Consider the approximation of (1.3) given by the following stochastic heat equation driven by a random potential:

$$
\left\{\begin{array}{l}
\frac{\partial}{\partial t} u^{\varepsilon, \tau}=\frac{1}{2} \Delta u^{\varepsilon, \tau}+u^{\varepsilon, \tau} \dot{B}^{\varepsilon, \tau}(t, x), \\
u^{\varepsilon, \tau}(0, x)=\varphi(x) .
\end{array}\right.
$$


By Fubini's theorem and (4.1) we can write

$$
\begin{aligned}
\int_{0}^{t} \dot{B}^{\varepsilon, \tau}\left(t-s, W_{s}^{x}\right) d s & =\int_{0}^{t} d s \int_{0}^{t} \int_{\mathbb{R}} \phi_{\tau}(t-s-r) p_{\varepsilon}\left(W_{s}^{x}-y\right) B(d r, d y) \\
& =\int_{0}^{t} \int_{\mathbb{R}}\left(\int_{0}^{t} \phi_{\tau}(t-s-r) p_{\varepsilon}\left(W_{s}^{x}-y\right) d s\right) B(d r, d y)=V^{\varepsilon, \tau}(t, x),
\end{aligned}
$$

where $V^{\varepsilon, \tau}(t, x)$ is defined by (3.8). It follows from the classical Feynman-Kac formula that

$$
\begin{aligned}
u^{\varepsilon, \tau}(t, x) & =E^{W}\left[\varphi\left(W_{t}^{x}\right) \exp \left\{\int_{0}^{t} \dot{B}^{\varepsilon, \tau}\left(t-s, W_{s}^{x}\right) d s\right\}\right] \\
& =E^{W}\left[\varphi\left(W_{t}^{x}\right) \exp \left(V^{\varepsilon, \tau}(t, x)\right)\right],
\end{aligned}
$$

where $W^{x}$ is a standard Brownian motion independent of $B$ and starting at $x$.

Lemma 4.1 Let $V(t, x)$ be given by (3.1). Define the process

$$
u(t, x)=E^{W}\left[\varphi\left(W_{t}^{x}\right) \exp (V(t, x))\right], \quad t \geq 0, x \in \mathbb{R} .
$$

Then we have

$$
\lim _{\varepsilon, \tau \downarrow 0} E^{B}\left|u^{\varepsilon, \tau}(t, x)-u(t, x)\right|^{p}=0
$$

for all $p \geq 2, x \in \mathbb{R}$, and $t \geq 0$.

Proof For all $p \geq 2, x \in \mathbb{R}$, and $t \geq 0$, we have

$$
\begin{aligned}
E^{B}\left|u^{\varepsilon, \tau}(t, x)-u(t, x)\right|^{p} & =E^{B}\left|E^{W}\left(\varphi\left(W_{t}^{x}\right)\left[\exp \left(V^{\varepsilon, \tau}(t, x)\right)-\exp (V(t, x))\right]\right)\right|^{p} \\
& \leq\|\varphi\|_{\infty}^{p} E\left|\exp \left(V^{\varepsilon, \tau}(t, x)\right)-\exp (V(t, x))\right|^{p}
\end{aligned}
$$

On the other hand, we have

$$
\begin{aligned}
E\left[\exp \left(\lambda V^{\varepsilon, \tau}(t, x)\right)\right] & =E \exp \left(\frac{1}{2} \lambda^{2}\left\|A^{\varepsilon, \tau}(t, x)\right\|_{\mathcal{H}}^{2}\right) \\
& \leq E \exp \left(\frac{1}{2} \lambda^{2} C \int_{0}^{t} \int_{0}^{t}|r-s|^{2 H K-2}\left|W_{r}-W_{s}\right|^{2 H^{\prime} K^{\prime}-2} d r d s\right)<\infty
\end{aligned}
$$

for all $\varepsilon, \tau>0$, which deduces, for any $\lambda \in \mathbb{R}$,

$$
\sup _{\varepsilon, \tau>0} E\left[\exp \left(V^{\varepsilon, \tau}(t, x)\right)\right]<\infty
$$

Combining this with the fact that

$$
\exp \left(V^{\varepsilon, \tau}(t, x)\right) \longrightarrow \exp (V(t, x))
$$

in probability, by Theorem 3.1 we obtain the lemma. 
Proof of Theorem 4.1 Let $f$ be a smooth function with compact support. Then we have

$$
\begin{aligned}
\int_{\mathbb{R}} u^{\varepsilon, \tau}(t, x) f(x) d x= & \int_{\mathbb{R}} \varphi(x) f(x) d x+\frac{1}{2} \int_{0}^{t} \int_{\mathbb{R}} u^{\varepsilon, \tau}(s, x) \Delta f(x) d x d s \\
& +\int_{0}^{t} \int_{\mathbb{R}} u^{\varepsilon, \tau}(s, x) f(x) \dot{B}^{\varepsilon, \tau}(s, x) d x d s
\end{aligned}
$$

almost surely for all $t \geq 0$. Therefore, to end the proof, we only need to prove that

$$
\int_{0}^{t} \int_{\mathbb{R}} u^{\varepsilon, \tau}(s, x) f(x) \dot{B}^{\varepsilon, \tau}(s, x) d x d s \rightarrow \int_{0}^{t} \int_{\mathbb{R}} u(s, x) f(x) B(d s, d x)
$$

in probability as $\varepsilon$ and $\tau$ tend to zero. It follows from Lemma 4.1 that the random variables of the right-hand side in (4.7) converges in $L^{2}$ to the random variable

$$
\Upsilon:=\int_{\mathbb{R}} u(t, x) f(x) d x-\int_{\mathbb{R}} f(x) \varphi(x) d x-\frac{1}{2} \int_{0}^{t} \int_{\mathbb{R}} u(s, x) \Delta f(x) d x d s
$$

as $\varepsilon$ and $\tau$ tend to zero. Denote

$$
\mathcal{C}^{\varepsilon, \tau}(t)=\int_{0}^{t} \int_{\mathbb{R}}\left[u^{\varepsilon, \tau}(s, x)-u(s, x)\right] f(x) \dot{B}^{\varepsilon, \tau}(s, x) d x d s, \quad t \geq 0,
$$

for all $\varepsilon, \tau>0$. Then, since $\lim _{\varepsilon, \tau \downarrow 0} \mathcal{C}^{\varepsilon, \tau}(t)=0$ in $L^{2},(4.7)$ implies that

$$
\int_{0}^{t} \int_{\mathbb{R}} u(s, x) f(x) \dot{B}^{\varepsilon, \tau}(s, x) d x d s=\int_{0}^{t} \int_{\mathbb{R}} u^{\varepsilon, \tau}(s, x) f(x) \dot{B}^{\varepsilon, \tau}(s, x) d x d s-\mathcal{C}^{\varepsilon, \tau}(t)
$$

converges to $\Upsilon$ in probability as $\varepsilon$ and $\tau$ tend to zero. So we have that $u(s, x) f(x)$ is Stratonovich integrable and

$$
\int_{0}^{t} \int_{\mathbb{R}} u(s, x) f(x) B(d s, d x)=\Upsilon .
$$

Thus, to end the proof, we only need to show that

$$
\mathcal{C}^{\varepsilon, \tau}(t) \longrightarrow 0
$$

in $L^{2}$ as $\varepsilon$ and $\tau$ tend to zero. Denote

$$
\psi^{\varepsilon, \tau}(r, z):=\int_{0}^{t} \int_{\mathbb{R}}\left[u^{\varepsilon, \tau}(s, x)-u(s, x)\right] f(x) \phi_{\tau}(s-r) p_{\varepsilon}(x-z) d x d s
$$

for all $\varepsilon, \tau>0, r \geq 0$, and $z \in \mathbb{R}$ and by $\delta^{B}\left(\psi^{\varepsilon, \tau}\right)=\int_{0}^{t} \int_{\mathbb{R}} \psi^{\varepsilon, \tau}(r, z) \delta B(r, z)$ the divergence or the Skorokhod integral $\psi^{\varepsilon, \tau}$. Then we have

$$
\begin{aligned}
\mathcal{C}^{\varepsilon, \tau}(t)= & \int_{0}^{t} \int_{\mathbb{R}} \psi^{\varepsilon, \tau}(r, z) \delta B(r, z) \\
& +\int_{0}^{t} \int_{\mathbb{R}} f(x)\left\langle D^{B}\left(u^{\varepsilon, \tau}(s, x)-u(s, x)\right), \phi_{\tau}(s-\cdot) p_{\varepsilon}(x-\cdot)\right\rangle_{\mathcal{H}} d x d s \\
\equiv & \mathcal{C}_{1}^{\varepsilon, \tau}(t)+\mathcal{C}_{2}^{\varepsilon, \tau}(t)
\end{aligned}
$$

for all $\varepsilon, \tau>0$ and $t \geq 0$, and the theorem follows from the next lemmas. 
Lemma 4.2 Let $f$ be a smooth function with compact support. Then $\mathcal{C}_{1}^{\varepsilon, \tau}(t)$ converges to zero in $L^{2}$ for all $t \geq 0$ as $\varepsilon$ and $\tau$ tend to zero.

Proof For the process $\mathcal{C}_{1}^{\varepsilon, \tau}$, we estimate

$$
E\left|\mathcal{C}_{1}^{\varepsilon, \tau}(t)\right|^{2} \leq E\left(\left\|\psi^{\varepsilon, \tau}\right\|_{\mathcal{H}}^{2}\right)+E\left\|D \psi^{\varepsilon, \tau}\right\|_{\mathcal{H} \otimes \mathcal{H}}^{2}
$$

by using the $L^{2}$-estimate for the Skorokhod integral.

Step I. We first have

$$
\begin{aligned}
E\left(\left\|\psi^{\varepsilon, \tau}\right\|_{\mathcal{H}}^{2}\right)= & \int_{0}^{t} \int_{\mathbb{R}} \int_{0}^{t} \int_{\mathbb{R}} E\left[u^{\varepsilon, \tau}(s, x)-u(s, x)\right]\left[u^{\varepsilon, \tau}(r, y)-u(r, y)\right] \\
& \cdot f(x) f(y)\left\langle\phi_{\tau}(s-\cdot) p_{\varepsilon}(x-\cdot), \phi_{\tau}(r-\cdot) p_{\varepsilon}(z-\cdot)\right\rangle_{\mathcal{H}} d x d s d y d r
\end{aligned}
$$

for all $\varepsilon, \tau>0$. Notice that

$$
\begin{aligned}
\left\langle\phi_{\tau}(s-\cdot) p_{\varepsilon}(x-\cdot), \phi_{\tau}(r-\cdot) p_{\varepsilon}(z-\cdot)\right\rangle_{\mathcal{H}} \\
=\kappa \int_{0}^{t} \int_{0}^{t} \phi_{\tau}(s-u) \phi_{\tau}(r-v)|u-v|^{2 H K-2} d v d u \\
\quad \cdot \int_{\mathbb{R}} \int_{\mathbb{R}} p_{\varepsilon}(x-z) p_{\varepsilon}(y-w)|z-w|^{2 H^{\prime} K^{\prime}-2} d z d w \\
\leq C|s-r|^{2 H K-2}|x-y|^{2 H^{\prime} K^{\prime}-2}
\end{aligned}
$$

for all $s, r \geq 0$ and $x, y \in \mathbb{R}$ by Lemma 3.2. We see that, as a consequence, the integrand on the right-hand side of (4.10) converges to zero as $\varepsilon$ and $\tau$ tend to zero for any $s, r \geq 0$ and $x, y \in \mathbb{R}$.

On the other hand, from the proof of Lemma 4.1 we have that

$$
E\left[\left|u^{\varepsilon, \tau}(s, x)\right|^{2}\right] \leq C<\infty
$$

for any $s, r \geq 0$ and $x, y \in \mathbb{R}$, which shows that the integrand on the right-hand side of (4.10) is bounded. Then, by the dominated convergence theorem we have that $E\left(\left\|\psi^{\varepsilon, \tau}\right\|_{\mathcal{H}}^{2}\right)$ converges to zero as $\varepsilon$ and $\tau$ tend to zero.

Step II. We next show that

$$
E\left\|D \psi^{\varepsilon, \tau}\right\|_{\mathcal{H} \otimes \mathcal{H}}^{2} \longrightarrow 0
$$

as $\varepsilon$ and $\tau$ tend to zero. Clearly, we have

$$
D u^{\varepsilon, \tau}(t, x)=E^{B}\left[f\left(W_{t}+x\right) \exp \left(V_{t, x}^{\varepsilon, \tau}\right) A^{\varepsilon, \tau}(t, x)\right]
$$

Let $W^{1}$ and $W^{2}$ be two independent Brownian motions. Then

$$
\begin{aligned}
& E\left\langle D u^{\varepsilon, \tau}(t, x), D u^{\varepsilon^{\prime}, \tau^{\prime}}(t, x)\right\rangle_{\mathcal{H}} \\
& =E^{B} E^{W_{1}, W_{2}}\left[f\left(W_{t}^{1}+x\right) f\left(W_{t}^{2}+x\right)\right. \\
& \left.\quad \cdot \exp \left\{V_{W^{1}}^{\varepsilon, \tau}(t, x)+V_{W^{2}}^{\varepsilon^{\prime}, \tau^{\prime}}(t, x)\right\}\left\langle A_{t, x}^{\varepsilon, \tau}\left(W^{2}\right), A_{t, x}^{\varepsilon^{\prime}, \tau^{\prime}}\left(W^{2}\right)\right\rangle_{\mathcal{H}}\right]
\end{aligned}
$$


where $E^{W_{1}, W_{2}}$ is the expectation with respect to $\left(W_{1}, W_{2}\right)$, and

$$
A_{t, x}^{\varepsilon, \tau}\left(W^{i}\right)=\int_{0}^{t} \phi_{\tau}(t-s-\cdot) p_{\varepsilon}\left(W_{s}^{i}+x-\cdot\right) d s
$$

and

$$
V_{W^{i}}^{\varepsilon, \tau}(t, x)=\int_{0}^{t} \int_{\mathbb{R}} A_{t, x}^{\varepsilon, \tau}\left(W^{i}\right)(r, y) B(d r, d y)
$$

for all $t \geq 0$ and $x \in \mathbb{R}$. Then, from the previous results we have

$$
\begin{aligned}
\lim _{\varepsilon, \tau \downarrow 0}\left\langle D u^{\varepsilon, \tau}(t, x),\left.D u^{\varepsilon, \tau}(t, x)\right|_{\mathcal{H}}\right. \\
=E\left[f\left(W_{t}^{1}+x\right) f\left(W_{t}^{2}+x\right)\right. \\
\quad \cdot \exp \left(\frac{1}{2} \kappa \sum_{j, k=1}^{2} \int_{0}^{t} \int_{0}^{t}|s-r|^{2 H K-2}\left|W_{s}^{j}-W_{r}^{k}\right|^{2 H^{\prime} K^{\prime}-2} d r d s\right) \\
\left.\quad \cdot \kappa \int_{0}^{t} \int_{0}^{t}|s-r|^{2 H K-2}\left|W_{s}^{1}-W_{r}^{2}\right|^{2 H^{\prime} K^{\prime}-2} d r d s\right],
\end{aligned}
$$

which implies that $u^{\varepsilon, \tau}(t, x)$ converges in the space $\mathbb{D}_{1,2}$ to $u(t, x)$ as $\varepsilon$ and $\tau$ tend to zero and

$$
E\left\|D \psi^{\varepsilon, \tau}\right\|_{\mathcal{H}}^{2} \leq C<\infty
$$

for all $\varepsilon, \tau>0, x \in \mathbb{R}$, and $s \in[0, t]$. It follows that

$$
\begin{aligned}
E\left\|D \psi^{\varepsilon, \tau}\right\|_{\mathcal{H} \otimes \mathcal{H}}^{2}= & \int_{0}^{t} \int_{\mathbb{R}} \int_{0}^{t} \int_{\mathbb{R}} f(x) f(y) \\
& \cdot E\left\langle D^{B}\left(u^{\varepsilon, \tau}(s, x)-u(s, x)\right), D^{B}\left(u^{\varepsilon, \tau}(r, y)-u(r, y)\right)\right\rangle_{\mathcal{H}} \\
& \cdot\left\langle\phi_{\tau}(s-\cdot) p_{\varepsilon}(x-\cdot), \phi_{\tau}(r-\cdot) p_{\varepsilon}(y-\cdot)\right\rangle_{\mathcal{H}} d x d s d y d r
\end{aligned}
$$

converges to zero as $\varepsilon$ and $\tau$ tend to zero. Hence, $\mathcal{C}_{1}^{\varepsilon, \tau}(t)$ converges to zero in $L^{2}$ as $\varepsilon$ and $\tau$ tend to zero, and the lemma follows.

Lemma 4.3 Let $f$ be a smooth function with compact support. Then $\mathcal{C}_{2}^{\varepsilon, \tau}(t)$ converges to zero in $L^{2}$ for all $t \geq 0$ as $\varepsilon$ and $\tau$ tend to zero.

Proof Denote

$$
\mathcal{C}_{2,1}^{\varepsilon, \tau}(t):=\int_{0}^{t} \int_{\mathbb{R}} f(x) E^{W}\left[\varphi\left(W_{s}^{x}\right) \exp \left(V^{\varepsilon, \tau}(s, x)\right)\left\langle A_{s, x}^{\varepsilon, \tau},\left.\phi_{\tau}(s-\cdot) p_{\varepsilon}(x-\cdot)\right|_{\mathcal{H}}\right] d x d s\right.
$$

and

$$
\mathcal{C}_{2,2}^{\varepsilon, \tau}(t):=\int_{0}^{t} \int_{\mathbb{R}} f(x) E^{W}\left[\varphi\left(W_{s}^{x}\right) \exp (V(s, x))\left\langle\delta\left(W_{s-}^{x}-\right), \phi_{\tau}(s-\cdot) p_{\varepsilon}(x-\cdot)\right\rangle_{\mathcal{H}}\right] d x d s
$$


for all $\varepsilon, \tau>0$ and $t \geq 0$. Then we can decompose $\mathcal{C}_{2}^{\varepsilon, \tau}(t)$ as

$$
\mathcal{C}_{2}^{\varepsilon, \tau}(t)=\mathcal{C}_{2,1}^{\varepsilon, \tau}(t)-\mathcal{C}_{2,2}^{\varepsilon, \tau}(t)
$$

for all $\varepsilon, \tau>0$ and $t \geq 0$. Clearly, by Lemma 3.2 we have

$$
\begin{aligned}
\left\langle A_{s, x}^{\varepsilon, \tau}, \phi_{\tau}(s-\cdot) p_{\varepsilon}(x-\cdot)\right\rangle_{\mathcal{H}}= & \kappa \int_{[0,]^{3}} \int_{\mathbb{R}^{2}}|r-v|^{2 H K-2}|y-z|^{2 H^{\prime} K^{\prime}-2} \phi_{\tau}(s-u) p_{\varepsilon}\left(W_{u}^{x}-y\right) \\
& \cdot \phi(s-v) p_{\varepsilon}(x-z) d y d z d r d v d u \\
\leq & C \int_{0}^{s} r^{2 H K-2}\left|W_{r}\right|^{2 H^{\prime} K^{\prime}-2} d r
\end{aligned}
$$

and

$$
\begin{aligned}
& \left\langle\delta\left(W_{s-}^{x}-\right), \phi_{\tau}(s-\cdot) p_{\varepsilon}(x-\cdot)\right\rangle_{\mathcal{H}} \\
& \quad=\kappa \int_{[0, s]^{2}} \int_{\mathbb{R}} v^{2 H K-2}\left|W_{r}^{x}-y\right|^{2 H^{\prime} K^{\prime}-2} \phi_{\tau}(r-v) p_{\varepsilon}(x-y) d y d v d r \\
& \quad \leq C \int_{0}^{s} r^{2 H K-2}\left|W_{r}\right|^{2 H^{\prime} K^{\prime}-2} d r
\end{aligned}
$$

for all $\varepsilon, \tau>0$ and $t \geq 0$. Notice that

$$
\int_{0}^{s} r^{2 H K-2}\left|W_{r}\right|^{2 H^{\prime} K^{\prime}-2} d r
$$

is square integrable for all $2 H K+H^{\prime} K^{\prime}>2$. In fact, we have

$$
\begin{aligned}
E\left(\int_{0}^{s} r^{2 H K-2}\left|W_{r}\right|^{2 H^{\prime} K^{\prime}-2} d r\right)^{2} & =\int_{0}^{s} \int_{0}^{r}(r v)^{2 H K-2} E\left|W_{r} W_{v}\right|^{2 H^{\prime} K^{\prime}-2} d r d v \\
& \leq C \int_{0}^{s} r^{2 H K+H^{\prime} K^{\prime}-3} \int_{0}^{r} v^{2 H K-2}(r-v)^{H^{\prime} K^{\prime}-1} d r d v \\
& =C s^{2\left(2 H K+H^{\prime} K^{\prime}\right)-4}
\end{aligned}
$$

It follows from the dominated convergence theorem that $\mathcal{C}_{2,1}^{\mathcal{\varepsilon}, \tau}(t)$ and $\mathcal{C}_{2,2}^{\varepsilon, \tau}(t)$ both converge in $L^{2}$ to

$$
\kappa \int_{0}^{t} \int_{\mathbb{R}} f(x) E^{W}\left(\varphi\left(W_{s}^{x}\right) \exp (V(s, x)) \int_{0}^{s} r^{2 H K-2}\left|W_{r}\right|^{2 H^{\prime} K^{\prime}-2} d r\right) d x d s
$$

for all $t \geq 0$ as $\varepsilon$ and $\tau$ tend to zero, which says that $\mathcal{C}_{2}^{\varepsilon, \tau}(t)$ converges to zero in $L^{2}$ as $\varepsilon$ and $\tau$ tend to zero. This completes the proof.

Theorem 4.2 Let $2 H^{\prime} K^{\prime}=1,2 H K>\frac{3}{2}$, and let $\varphi$ be a bounded measurable function. Then the process

$$
u(t, x)=E^{W}\left[\varphi\left(W_{t}^{x}\right) \exp \left\{\int_{0}^{t} \int_{\mathbb{R}} \delta\left(W_{t-r}^{x}-y\right) B(d r, d y)\right\}\right]
$$


is a weak solution to (1.3), where $E^{W}$ denotes the expectation with respect to the Brownian motion $W_{t}^{x}$, and $\delta$ denotes the Dirac delta function.

Corollary 4.1 Let $2 H K, 2 H^{\prime} K^{\prime} \geq 1$ and $2 H K+H^{\prime} K^{\prime}>2$. Then the solution

$$
u(t, x)=E^{W}\left[\varphi\left(W_{t}^{x}\right) \exp \left\{\int_{0}^{t} \int_{\mathbb{R}} \delta\left(W_{t-r}^{x}-y\right) B(d r, d y)\right\}\right]
$$

has finite moments of all orders.

Recall that an $\mathscr{F}_{t}$-adapted $L^{p}(\mathbb{R})$-valued stochastic process $u:[0, T] \times \mathbb{R} \rightarrow u(t, x, \omega) \in \mathbb{R}$ is a mild solution to SPDE (1.3) for any $T>0$ if $u(t, x)$ satisfies the integral equation

$$
u(t, x)=\int_{\mathbb{R}} G(t ; x, y) \varphi(y) d y+\int_{0}^{t} \int_{\mathbb{R}} G(t-s ; x-y) u(s, y) B^{H}(d s, d y)
$$

for each $t \in[0, T]$, where $G(t-s ; x, y)$ denotes the heat kernel, that is, the fundamental solution of the heat equation

$$
\frac{\partial u}{\partial t}(t, x)=\Delta u(t, x)
$$

Moreover, we say that the uniqueness of (1.3) holds if whenever $u_{1}$ and $u_{2}$ are any two solutions to (1.3) with the same initial value, then $u_{1}(t, x)=u_{2}(t, x)$ a.s. for all $t \in[0, T]$ and $x \in \mathbb{R}$.

Theorem 4.3 Let $2 H K, 2 H^{\prime} K^{\prime} \geq 1$ and $2 H K+H^{\prime} K^{\prime}>2$. If $\varphi$ is a bounded measurable function, then the process

$$
u(t, x)=E^{W}\left[\varphi\left(W_{t}^{x}\right) \exp \left\{\int_{0}^{t} \int_{\mathbb{R}} \delta\left(W_{t-r}^{x}-y\right) B(d r, d y)\right\}\right]
$$

is a mild solution to (1.3), where $E^{W}$ denotes the expectation with respect to the Brownian motion $W_{t}^{x}$, and $\delta$ denotes the Dirac delta function.

\section{Regularity of the weak solution}

In this section, we give the Hölder continuity of the solution of (1.3) and show that the probability law of the solution admits a smooth density by using the Feynman-Kac formula established in the previous section.

Theorem 5.1 Let $2 H K, 2 H^{\prime} K^{\prime}>1,2 H K+H^{\prime} K^{\prime}>2$, and let $u(t, x)$ be the solution of (1.3). Then $(t, x) \mapsto u(t, x)$ is Hölder continuous with order $v \in\left(0, \frac{1}{2}\left(2 H K+H^{\prime} K^{\prime}-2\right)\right)$ in time $t$ and $x$, that is, for any $T, M>0$, there is a positive random variable $K_{T, M}$ such that almost surely, for any $t, s \in[0, T]$ and $x, y \in[-M, M]$, we have

$$
|u(t, y)-u(s, x)| \leq K_{T, M}\left(|t-s|^{v}+|x-y|^{v}\right) .
$$

Proof Let $p \geq 2$. Notice that

$$
u(t, x)=E^{W}\left[\varphi\left(W_{t}^{x}\right) e^{V(t, x)}\right]
$$


for all $t \geq 0$ and $x \in \mathbb{R}$, where $V(t, x)$ is given by (3.1). Then

$$
\begin{aligned}
E^{B}|u(t, y)-u(s, x)|^{p}= & E^{B}\left|E^{W}\left(\varphi\left(W_{t}^{y}\right) e^{V(t, y)}-\varphi\left(W_{s}^{x}\right) e^{V(s, x)}\right)\right|^{p} \\
\leq & C E^{B}\left|E^{W} \varphi\left(W_{t}^{y}\right)\left(e^{V(t, y)}-e^{V(s, x)}\right)\right|^{p} \\
& +C E^{B}\left|E^{W} e^{V(s, x)}\left(\varphi\left(W_{t}^{y}\right)-\varphi\left(W_{s}^{x}\right)\right)\right|^{p} \\
\leq & C E^{B}\left|E^{W}\left(e^{V(t, y)}-e^{V(s, x)}\right)\right|^{p} \\
& +C|y-x|^{p} E^{B}\left|E^{W} e^{V(s, x)}\right|^{p}
\end{aligned}
$$

since $\varphi$ is bounded and the function

$$
x \mapsto E^{W}\left[\varphi\left(W_{t}^{x}\right)\right]=E^{W}\left[\varphi\left(W_{t}+x\right)\right]
$$

is $C^{\infty}$. Thus, we need only to estimate

$$
E^{B}\left|E^{W}\left(e^{V(t, y)}-e^{V(s, x)}\right)\right|^{p}
$$

To see this, we have

$$
\begin{aligned}
E^{B} & \left|E^{W}\left(e^{V(t, y)}-e^{V(s, x)}\right)\right|^{p} \\
& \leq E^{B}\left|E^{W}\left([V(t, y)-V(s, x)] e^{V(t, y) \vee V(s, x)}\right)\right|^{p} \\
& \leq E^{B}\left(\left(E^{W}\left([V(t, y)-V(s, x)]^{2}\right)\right)^{p / 2}\left(E^{W} e^{2(V(t, y) \vee V(s, x))}\right)^{p / 2}\right) \\
& \leq\left(E^{B}\left(E^{W}\left([V(t, y)-V(s, x)]^{2}\right)\right)^{p}\right)^{1 / 2}\left(E^{B}\left(E^{W} e^{2(V(t, y) \vee V(s, x))}\right)^{p}\right)^{1 / 2}
\end{aligned}
$$

by Cauchy's inequality. By the equivalence between the $L^{2}$-norm and the $L^{p}$-norm for a Gaussian random variable, Minkowski's inequality, and the exponential integrability, we can get

$$
\left(E^{W} e^{2(V(t, y) \vee V(s, x))}\right)^{p} \leq E^{W} e^{2 p(V(t, y) \vee V(s, x))} \leq C_{T, M}<\infty
$$

for all $|x|,|y| \leq M$ and $s, t \in[0, T]$. Consequently, the theorem follows from the estimate

$$
\begin{aligned}
E^{B}|u(t, y)-u(s, x)|^{p} & \leq C\left(E^{B}\left(E^{W}\left([V(t, y)-V(s, x)]^{2}\right)\right)^{p}\right)^{1 / 2} \\
& \leq C\left(E^{W} E^{B}[V(t, y)-V(s, x)]^{2}\right)^{p / 2}
\end{aligned}
$$

and the next lemma.

Lemma 5.1 Let $V(t, x)$ be given by (3.1), and let $T, M>0$. Then we have

$$
E^{B}\left([V(t, y)-V(s, x)]^{2}\right) \leq C\left(|t-s|^{2 H K+H^{\prime} K^{\prime}-2}+|x-y|^{2 H K+H^{\prime} K^{\prime}-2}\right)
$$

for all $t, s \in[0, T]$ and $x, y \in[-M, M]$, where $C>0$ is a constant depending only on $T$ and $M$. 
Proof We have

$$
\begin{aligned}
E^{B}[V(t, y)-V(s, x)]^{2}= & \kappa E^{W}\left(\int_{0}^{s} \int_{0}^{s}|r-v|^{2 H K-2}\left|W_{s-r}-W_{s-\nu}\right|^{2 H^{\prime} K^{\prime}-2} d r d v\right. \\
& +\int_{0}^{t} \int_{0}^{t}|r-v|^{2 H K-2}\left|W_{t-r}-W_{t-v}\right|^{2 H^{\prime} K^{\prime}-2} d r d v \\
& \left.-2 \int_{0}^{s} \int_{0}^{t}|r-v|^{2 H K-2}\left|W_{s-r}-W_{t-v}+x-y\right|^{2 H^{\prime} K^{\prime}-2} d r d v\right) \\
\equiv & \Phi(s, t ; x, y)
\end{aligned}
$$

for all $t>s \geq 0$ and $x>y$. Now, in order to end the proof, we need only to estimate $\Phi(s, t ; x, y)$.

Step I. We estimate $\Phi(t, t ; x, y)$ for all $t \in[0, T]$ and $M \geq x>y \geq-M$. We have

$$
\begin{aligned}
\Phi(t, t ; x, y)= & 2 \kappa \int_{0}^{t} \int_{0}^{t}|r-v|^{2 H K-2} \\
& \cdot E\left(\left|W_{t-r}-W_{t-v}\right|^{2 H^{\prime} K^{\prime}-2}-\left|W_{t-r}-W_{t-v}+x-y\right|^{2 H^{\prime} K^{\prime}-2}\right) d r d v \\
= & 2 \kappa \int_{0}^{t} \int_{0}^{t}|r-v|^{2 H K+H^{\prime} K^{\prime}-3} E\left(|\xi|^{2 H^{\prime} K^{\prime}-2}-\left|\xi+\frac{x-y}{\sqrt{|r-v|}}\right|^{2 H^{\prime} K^{\prime}-2}\right) d r d v,
\end{aligned}
$$

where $\xi$ denotes a standard normal variable. An elementary calculation shows that (see Hu et al. [12])

$$
E\left(|\xi|^{-\alpha}-|\xi+w|^{-\alpha}\right) \leq C \min \left\{1, w^{2}+w^{3-\alpha}\right\}
$$

with $0<\alpha<1$ and $w \geq 0$, which gives

$$
\begin{aligned}
\Phi(t, t ; x, y) & \leq C \int_{D_{1}}|r-v|^{2 H K+H^{\prime} K^{\prime}-3} d r d v+C \int_{D_{2}}|r-v|^{2 H K+H^{\prime} K^{\prime}-3} \frac{(x-y)^{2}}{|r-v|} d r d v \\
& \leq C \int_{D_{1}}|r-v|^{2 H K+H^{\prime} K^{\prime}-3} d r d v+C(x-y)^{1+\beta} \int_{D_{2}}|r-v|^{2 H K+H^{\prime} K^{\prime}-3-\beta} d r d v \\
& \leq C(x-y)^{2 H K+H^{\prime} K^{\prime}-2},
\end{aligned}
$$

where $D_{1}=\{(r, v)|0 \leq r, v \leq t ;| r-v \mid \leq x-y\}$ and $D_{2}=[0, t]^{2}-D_{1}$.

Step II. We estimate $\Phi(s, t ; x, x)$ for all $0 \leq s<t \leq T$ and $x \in[-M, M]$. We have

$$
\begin{aligned}
\Phi(s, t ; x, x)= & \kappa\left(\int_{0}^{s} \int_{0}^{s}|r-v|^{2 H K+H^{\prime} K^{\prime}-3} d r d v+\int_{0}^{t} \int_{0}^{t}|r-v|^{2 H K+H^{\prime} K^{\prime}-3} d r d v\right. \\
& \left.-2 \int_{0}^{s} \int_{0}^{t}|r-v|^{2 H K-2}|t-v-s+r|^{H^{\prime} K^{\prime}-1} d r d v\right) \\
= & \kappa \int_{s}^{t} \int_{s}^{t}|r-v|^{2 H K+H^{\prime} K^{\prime}-3} d r d v \\
& +2 \kappa \int_{0}^{s} \int_{0}^{t}|r-v|^{2 H K-2}\left(|r-v|^{H^{\prime} K^{\prime}-1}-|(t-s)+(r-v)|^{H^{\prime} K^{\prime}-1}\right) d r d v \\
\equiv & 2 \kappa\left(\Lambda_{1}(s, t)+\Lambda_{2}(s, t)\right) .
\end{aligned}
$$


Clearly, the first integral $\Lambda_{1}(s, t)$ equals $C|t-s|^{2 H K+H^{\prime} K^{\prime}-1}$. For the second integral $\Lambda_{2}(s, t)$, by the substitution

$$
u=r-v, w=v
$$

we have

$$
\begin{aligned}
\Lambda_{2}(s, t) & =\int_{0}^{s} \int_{0}^{t}|r-v|^{2 H K-2}\left(|r-v|^{H^{\prime} K^{\prime}-1}-|(t-s)+(r-v)|^{H^{\prime} K^{\prime}-1}\right) d r d v \\
& =\int_{0}^{t} d w \int_{-t}^{s}|u|^{2 H K-2}\left(|u|^{H^{\prime} K^{\prime}-1}-|(t-s)+u|^{H^{\prime} K^{\prime}-1}\right) d u \\
& =t \int_{-t}^{s}|u|^{2 H K-2}\left(|u|^{H^{\prime} K^{\prime}-1}-|(t-s)+u|^{H^{\prime} K^{\prime}-1}\right) d u \\
& \leq C(t-s)^{2 H K+H^{\prime} K^{\prime}-2},
\end{aligned}
$$

which implies

$$
\Phi(s, t ; x, x) \leq C(t-s)^{2 H K+H^{\prime} K^{\prime}-2}
$$

for all $0 \leq s<t \leq T$ and $x \in \mathbb{R}$.

Thus, we have obtained estimate (5.1).

Theorem 5.2 Let $2 H^{\prime} K^{\prime}=1,2 H K>\frac{3}{2}$, and let $u(t, x)$ be the solution of (1.3). Then $(t, x) \mapsto$ $u(t, x)$ is Hölder continuous with order $v \in\left(0, \frac{1}{2}\left(2 H K-\frac{3}{2}\right)\right)$ in time $t$ and $x$, that is, for any $T, M>0$, there is a positive random variable $K_{T, M}$ such that almost surely, for any $t, s \in[0, T]$ and $x, y \in[-M, M]$, we have

$$
|u(t, y)-u(s, x)| \leq K_{T, M}\left(|t-s|^{\nu}+|x-y|^{\nu}\right)
$$

Now, we show that the probability law of the solution $u(t, x)$ of $(1.3)$ has a smooth density with respect to the Lebesgue measure for any $t$ and $x$. To simplify, we let $\varphi(x) \equiv 1$. It follows that

$$
u(t, x)=E^{W}\left[e^{V_{W}(t, x)}\right]
$$

for any $t$ and $x$, where

$$
V_{W}(t, x)=\int_{0}^{t} \int_{\mathbb{R}} \delta\left(W_{t-r}^{x}-y\right) B(d r, d y) .
$$

Theorem 5.3 Suppose that $2 H K, 2 H^{\prime} K^{\prime} \geq 1,2 H K+H^{\prime} K^{\prime}>2$. Fix $t>0$ and $x \in \mathbb{R}$. Then, the law of $u(t, x)$ has a smooth density.

Proof We first prove the theorem for $2 H K, 2 H^{\prime} K^{\prime}>1$. Clearly, the Malliavin derivative of the solution is

$$
D_{r, y}^{B} u(t, x)=E^{W}\left[e^{V_{W}(t, x)} \delta\left(W_{t-r}^{x}-y\right)\right]
$$


By the general criterion for the smoothness of densities (see Nualart [8]) we only need to show that $\left\|D^{B}(t, x)\right\|_{\mathcal{H}}$ has negative moments of all orders for any $t>0$ and $x \in \mathbb{R}$, that is,

$$
E\left(\left\|D^{B}(t, x)\right\|_{\mathcal{H}}^{2 p}\right)<\infty
$$

for all $p>0, t>0$, and $x \in \mathbb{R}$. We have

$$
\begin{aligned}
\left\|D^{B}(t, x)\right\|_{\mathcal{H}}^{2} & =E^{W}\left[e^{V_{W_{1}}(t, x)+V_{W_{2}}(t, x)}\left\langle\delta\left(W_{t-r}^{1, x}-y\right), \delta\left(W_{t-r}^{2, x}-y\right)\right\rangle_{\mathcal{H}}\right] \\
& =\kappa E^{W}\left[e^{V_{W_{1}}(t, x)+V_{W_{2}}(t, x)} \int_{0}^{t} \int_{0}^{t}|r-s|^{2 H K-2}\left|W_{t-r}^{1}-W_{t-s}^{2}\right|^{2 H^{\prime} K^{\prime}-2} d s d r\right]
\end{aligned}
$$

for any $t>0$ and $x \in \mathbb{R}$, where $W^{1}$ and $W^{2}$ are independent Brownian motions. Using Jensen's inequality and Hölder's inequality, we obtain

$$
\begin{aligned}
\left\|D^{B}(t, x)\right\|_{\mathcal{H}}^{-2 p} \leq & \kappa^{-p} E\left[e^{-p\left[V_{W_{1}}(t, x)+V_{W_{2}}(t, x)\right]}\right. \\
& \left.\cdot\left(\int_{0}^{t} \int_{0}^{t}|r-s|^{2 H K-2}\left|W_{t-r}^{1}-W_{t-s}^{2}\right|^{2 H^{\prime} K^{\prime}-2} d s d r\right)^{-p}\right] \\
\leq & \kappa^{-p}\left[E e^{-p p_{1}\left[V_{W_{1}}(t, x)+V_{W_{2}}(t, x)\right]}\right] \frac{1}{p_{1}} \\
& \cdot\left[E\left(\int_{0}^{t} \int_{0}^{t}|r-s|^{2 H K-2}\left|W_{t-r}^{1}-W_{t-s}^{2}\right|^{2 H^{\prime} K^{\prime}-2} d s d r\right)^{-p p_{2}}\right]^{\frac{1}{p_{2}}}
\end{aligned}
$$

for any $t>0, x \in \mathbb{R}, p>0$, and $p_{1}, p_{2}>1$ with $\frac{1}{p_{1}}+\frac{1}{p_{2}}=1$. Now, let us estimate the final two terms. From Theorem 3.2 we have

$$
E e^{-\lambda\left[V_{W_{1}}(t, x)+V_{W_{2}}(t, x)\right]}<\infty
$$

for all $\lambda>0$. Moreover, by Jensen's inequality again, we have

$$
\begin{aligned}
& E\left(\int_{0}^{t} \int_{0}^{t}|r-s|^{2 H K-2}\left|W_{t-r}^{1}-W_{t-s}^{2}\right|^{2 H^{\prime} K^{\prime}-2} d s d r\right)^{-q} \\
& \quad \leq t^{-2 q-2} E \int_{0}^{t} \int_{0}^{t}|r-s|^{q(2-2 H K)}\left|W_{t-r}^{1}-W_{t-s}^{2}\right|^{\left(2-2 H^{\prime} K^{\prime} q\right)} d s d r \\
& \quad=t^{-2 q-2} \int_{0}^{t} \int_{0}^{t}|r-s|^{q(2-2 H K)} E\left|W_{t-r}^{1}-W_{t-s}^{2}\right|^{\left(2-2 H^{\prime} K^{\prime}\right) q} d s d r \\
& =t^{-2 q-2} E|\xi|^{q\left(2-2 H^{\prime} K^{\prime}\right)} \int_{0}^{t} \int_{0}^{t}|r-s|^{q(2-2 H K)}|2 t-r-s|^{\left(1-H^{\prime} K^{\prime}\right) q} d s d r \\
& \quad=C t^{q\left(1-2 H K-H^{\prime} K^{\prime}\right)}<\infty
\end{aligned}
$$

for any $t>0$ and $q>0$. Similarly, we can prove the theorem for $2 H^{\prime} K^{\prime}=1$ and $2 H K>1$. This completes the proof. 
Authors' contributions

All authors participated in drafting, revising, and commenting the manuscript. All authors read and approved the final manuscript.

\section{Author details}

${ }^{1}$ College of Information Science and Technology, Donghua University, 2999 North Renmin Rd., Songjiang, Shanghai, 201620, P.R. China. '2Department of Mathematics and Physics, Bengbu University, 1866 Caoshan Rd., Bengbu, 233030, P.R. China. ${ }^{3}$ Department of Mathematics, College of Science, Donghua University, 2999 North Renmin Rd., Songjiang, Shanghai, 201620, P.R. China.

\section{Acknowledgements}

The Project-sponsored by NSFC (11171062, 11426036), Innovation Program of Shanghai Municipal Education Commission (12ZZ063), Natural Science Foundation of Anhui Province (1408085QA10), Key Program in the Youth Elite Support Plan in Universities of Anhui Province (gxyqZD2016354), Quality project of Anhui Province (2015jyxm386) and Key Natural Science Foundation of Anhui Education Commission (KJ2016A453).

Received: 16 December 2015 Accepted: 10 March 2016 Published online: 21 March 2016

\section{References}

1. Alós, E, Mazet, O, Nualart, D: Stochastic calculus with respect to Gaussian processes. Ann. Probab. 29, 766-801 (2001)

2. Biagini, F, Hu, Y, Øksendal, B, Zhang, T: Stochastic Calculus for $\mathrm{fBm}$ and Applications. Probability and Its Application. Springer, Berlin (2008)

3. Decreusefond, L, Üstünel, AS: Stochastic analysis of the fractional Brownian motion. Potential Anal. 10, 177-214 (1999)

4. Gradinaru, M, Nourdin, I, Russo, F, Vallois, P: $m$-Order integrals and generalized Itô's formula; the case of a fBm with any Hurst index. Ann. Inst. Henri Poincaré Probab. Stat. 41, 781-806 (2005)

5. Hu, Y: Integral transformations and anticipative calculus for fractional Brownian motions. Mem. Am. Math. Soc. 175, 825 (2005)

6. Mishura, YS: Stochastic Calculus for Fractional Brownian Motion and Related Processes. Lect. Notes in Math., vol. 1929 (2008)

7. Nourdin, I: Selected Aspects of Fractional Brownian Motion. Springer, Berlin (2012)

8. Nualart, D: Malliavin Calculus and Related Topics, 2nd edn. Springer, New York (2006)

9. Tudor, CA: Analysis of Variations for Self-Similar Processes. Springer, Berlin (2013)

10. Houdré, C, Villa, J: An example of infinite dimensional quasi-helix. In: Stochastic Models (Mexico City, 2002). Contemp. Math., vol. 336, pp. 195-201. Am. Math. Soc., Providence (2003)

11. Freidlin, M: Functional Integration and Partial Differential Equations. Annals of Mathematics Studies, vol. 109. Princeton University Press, Princeton (1985)

12. $\mathrm{Hu}, \mathrm{Y}$, Nualart, D, Song, J: Feynman-Kac formula for heat equation driven by fractional white noise. Ann. Probab. 39 291-326 (2011)

13. $\mathrm{Hu}, \mathrm{Y}, \mathrm{Lu}, \mathrm{F}$, Nualart, D: Feynman-Kac formula for the heat equation driven by fractional noise with Hurst parameter $H<1 / 2$. Ann. Probab. 40, 1041-1068 (2012)

14. Es-sebaiy, K, Tudor, CA: Multidimensional bi-fractional Brownian motion: Itô and Tanaka formulas. Stoch. Dyn. 7 , 366-388 (2007)

15. Jiang, Y, Wang, Y: Self-intersection local times and collision local times of bi-fractional Brownian motions. Sci. China Ser. A 52, 1905-1919 (2009)

16. Kruk, I, Russo, F, Tudor, CA: Wiener integrals, Malliavin calculus and covariance measure structure. J. Funct. Anal. 249, 92-142 (2007)

17. Lei, P, Nualart, D: A decomposition of the bi-fractional Brownian motion and some applications. Stat. Probab. Lett. 79 619-624 (2009)

18. Russo, F, Tudor, CA: On the bi-fractional Brownian motion. Stoch. Process. Appl. 5, 830-856 (2006)

19. Tudor, CA, Xiao, Y: Some path properties of bi-fractional Brownian motion. Bernoulli 13, 1023-1052 (2007)

20. Shen, G, Yan, L: Smoothness for the collision local times of bi-fractional Brownian motions. Sci. China Math. 54, 1859-1873 (2011)

21. Yan, L, Liu, J, Chen, C: On the collision local time of bi-fractional Brownian motions. Stoch. Dyn. 9, 479-491 (2009)

22. Yan, L, Gao, B, Liu, J: The Bouleau-Yor identity for a bi-fBm. Stochastics 86, 382-414 (2014)

23. Chen, X: Random Walk Intersections: Large Deviations and Related Topics. Mathematical Surveys and Monographs, vol. 157. Am. Math. Soc., Providence (2009)

24. Russo, F, Vallois, P: Forward, backward and symmetric stochastic integration. Probab. Theory Relat. Fields 97, 403-421 (1993) 